\title{
How Future Depends on Past and Rare Events in Systems of Life ${ }^{1}$
}

\author{
Giuseppe Longo \\ Centre Cavaillès, République des Savoirs, \\ CNRS, Collège de France et Ecole Normale Supérieure, Paris, \\ and Department of Integrative Physiology and Pathobiology, \\ Tufts University School of Medicine, Boston. \\ http://www.di.ens.fr/users/longo
}

\begin{abstract}
The dependence on history of both present and future dynamics of life is a common intuition in biology and in humanities. Historicity will be understood in terms of changes of the space of possibilities (or of "phase space") as well as by the role of diversity in life's structural stability and of rare events in history formation. We hint to a rigorous analysis of "path dependence" in terms of invariants and invariance preserving transformations, as it may be found also in physics, while departing from the physico-mathematical analyses. The idea is that the (relative or historicized) invariant traces of the past under organismal or ecosystemic transformations contribute to the understanding (or the "theoretical determination") of present and future states of affairs. This yields a peculiar form of unpredictability (or randomness) in biology, at the core of novelty formation: the changes of observables and pertinent parameters may depend also on past events. In particular, in relation to the properties of synchronic measurement in physics, the relevance of diachronic measurement in biology is highlighted. This analysis may a fortiori apply to cognitive and historical human dynamics, while allowing to investigate some general properties of historicity in biology.
\end{abstract}

\section{1 - Introduction}

\section{1 - Main Theses}

Biology sits in between the mountain of the physico-mathematical construction and the depth of the investigations in human historical sciences. By the experimental methods and the nature of observation, it is a science of nature, yet the relevance of history in its understanding opens the way to the peculiar analyses proper to historical disciplines, beginning with the relevance of knowledge (and measurement) of past events.

In this paper, we highlight the following properties that allow to investigate the role of history in a specific knowledge construction, the sciences of life:

- the time dynamics include changes of the space of possibilities (or phase space ${ }^{2}$ )

- variability and diversity of observable objects is a component of the structural stability of the pertinent objects and their dynamics.

For our analysis, the pertinent biological observables are phenotypes, that is organisms' observable characteristics.

As a consequence of this approach to historicity we claim that, in biology:

- rare events crucially contribute to history.

To appear in Foundations of Science, 2017. This work is part of the project "Lois des dieux, des hommes et de la nature” at IEA-Nantes. A very preliminary version in Italian appeared in Paradigmi, XXXIII, Mag-Agosto, 2015.

2 This is the space of all pertinent parameters and observables, as measurable quantities (see below for more on this notion, which, in physics, is a precise, mathematical frame for the "space of all possibles dynamics"). 
In this perspective, a distinction will be made between:

- time of processes vs. time of history,

as different observable times in the same physical dimension ${ }^{3}$. In a sense, the proper historical time is scanned by rare events and changes of the phase space.

This analysis will allow to discuss the dependence of present (and future) phenomena on past events in systems of life. History dependence will be understood also on the grounds of measurement as the scientific form of access to phenomena, including past phenomena. The characteristics of measurement result from theoretical hypotheses, such as the choice of observables and parameters, and, in biology, from the historicity of their dynamics. In philosophical terms, this will be viewed as an epistemic issue, in reference to knowledge of history in terms of knowledge or measurement of past events, and, in particular, by a distinction between

- synchronic measurements vs. diachronic measurements (as access to past histories).

Finally, the physico-mathematical notion of invariance, joint to the transformations that characterize it ${ }^{4}$, will be used, in biology, in the novel sense of

- historicized invariance,

that we will gradually specify ${ }^{5}$.

\section{2 - Determination and Path Dependence, from Physics to Biology}

History is irrelevant in most existing physical theories. However, in sect. 2, we will briefly discuss the key role of historical analysis, in cosmology and, more pertinently, "path dependence" in Riemann's manifold (by Levi-Civita's parallel transport) as well as in cascades of singularities and critical transitions. Some informal references will be also made to how statistical physics and condensed matter physics describe path dependent phenomena.

The very interesting cases of "history dependence", or, more precisely, process dependence, in physics just quoted, and further discussed below, may help in the transitions from the theories of the inert to theories in biology. In (Longo, Montévil, 2014) we insisted on the possibility of this complex conceptual transition by focusing on an extension of the notion of critical phase transition. For example, physical theories of criticality are widely used in theoretical biology, since the '80s, and the reader may refer to (Longo, Montévil, 2014) for "extended criticality" in biology and references to "criticality" in physics. Others consider statistical physics and condensed matter physics as a possible theoretical junction between physics and biology (see (Goldenfeld, Woese, 2011) for an insightful survey). Biological Theories, however, are or may need to be at least as different from existing physical ones, as Relativity and Hydrodynamics are far from Quantum Mechanics (well ... these three theories present even physico-mathematical incompatibilities). Some theories of the inert may be enlightening for biology, in particular by suitable formal extensions, such as extended criticality or a two dimensional theory of biological time, where time acts as an operator (see (Bailly, Longo, 2011) and chapters 3 and 9 of (Longo, Montévil, 2014)). These extensions may help to shed light on life and its dynamics - and, if we are more lucky than physicists, there may be no mathematical incompatibility, like in the cases mentioned above, with existing physical theories.

In contrast to the role we attribute to history in biology (or in humanities), the current state of affairs is, in principle, sufficient for the understanding of the present of a physical dynamics, or, more rigorously, the "determination" of the intended system, also for the analysis of prediction may it be in terms of probabilities. That is, the "complete determination" of a physical system, within the exceptions we will discuss in sect. 2, is in principle independent on "how it got there", if

Cf., in physics, kinetic and potential energy are different observables in the same dimension, energy.

4 An "invariant" is characterized by the transformations that preserve it - typically, transformations in space and time as reference systems, like in Galileo's or Einstein's relativity, or, in mathematical terms, by groups or categorytheoretic isomorphisms.

5 Pierre Musso suggested the adjective "historicized" in order to express our concern for a conceptual transfer of the notion of invariance from a physico-mathematical frame to an historical science. 
the trajectory unfolds in a suitable phase space. Or, physical dynamics are analyzed as "State Determined Systems" - provided that the state or phase space is broadly construed. In short, also in those areas of physics where the notion of "path dependence" or even "history" occur and the response of a system to a changing context may depend on the history, the main effort of the theoretician is to invent a suitable phase or state space in order to obtain a "state determination". Moreover, in principle, the set of possible responses may be integrated in the system description and, if random, be given in terms of probabilities, see sect. 2 . We claim that, also in these cases, one develops an analysis of a time of processes, not of a proper historical time.

Our purpose here is to propose some sufficiently robust criteria to single out the peculiarity of a historical science, such as biology and biological evolution in particular, along the lines summarized in sect. 1.1. This will provide some conceptual tools to analyze the role of past histories in current and future dynamics of life systems and, by this, how history contributes to their peculiar form of intrinsic unpredictability. We claim that knowledge of past functions of biological structures is essential to the understanding of present and future dynamics and this on the grounds of their historical construction. We will then discuss the role of history in Darwin's Evolution or in human cognition, for the purposes of prediction, and stress the peculiar way one needs to theorize about it, well beyond, but, a priori, not incompatibly with, the rich attempts in physics.

The reader should be well aware that, in physics, "determinism" as mathematical determination does not mean predictability. Since Poincaré we know that even a simple set of (non-linear) equations or evolution functions, as a sound formal determination of a physical process (such as the Sun and two planets), does not imply its mathematical predictability (Poincaré's Three Body Problem, 1892, and non-linear classical systems: an immense literature there after, see (Devaney, 1989) for a classic textbook). Quantum-Mechanics reinforced this attitude by analyzing the dynamics of a quanton as the determination of an amplitude (a law) of probability (Schrödinger equation). This is a peculiar (and fantastic) way to integrate unpredictability, as randomness, in the theory, by the use of a possibly infinite dimensional (Hilbert) space as phase space. Jointly to the indetermination or non-commutativity of measurement (in short, knowledge of position and momentum, or the difference of their commuted measurements, cannot go below Planck's h), this gives the intrinsic (to the theory) nature of unpredictability and randomness in Quantum Mechanics ${ }^{6}$.

However, in all these systems, a pre-given phase space allows to define equations or evolution functions on the grounds of the (relative) knowledge of the present - and the complex case mentioned above, such as statistical physics, do not modify this robust role of states' determination in physics. Conversely, in order to write equations one needs to fix the pertinent phase space, that is the parameters and the observables, as well as the scale or level of description. Finally, note that also balance equations for, possibly stationary, far from equilibrium systems, are given in predefined phase spaces, (Nicolis, Prigogine, 1977; Vulpiani et al., 2014; Bertini et al., 2015).

\section{3 - Law-like processes}

6 On unpredictability and randomness. We stress that randomness, for us, means unpredictability in the intended theory (Calude, Longo, 2015). This relativizes randomness to the theory and its symmetries (Longo, Montévil, 2015). Without an at least tentative theoretical frame, one cannot talk of unpredictability: in order to "(un-)pre-dict" one needs to try first to "say” something (“dicere”, in latin). In this understanding, classical and quantum randomness differ, first since the two theories give non-classical probability values to entangled events (Einstein et al., 1935), (Aspect et al., 1982), second, because, by measurement and Schrödinger's equation, randomness is “integrated” (is intrinsic) to Quantum Mechanics. Moreover, but this is a further issue, classically any event has a cause, may it be a non-measurable fluctuation or perturbation, while in the current interpretation, some quantum random events may be a-causal, such as the spin up or down of an electron. Hidden variable theories claim that there is always a hidden cause; but then they get into different troubles: they need non-local variables in order to handle entanglement, a physico-mathematical inconsistency. In short, randomness is diverse and theory dependent in physics; a further notion of randomness is proposed here for biology (comparative analyses are presented in (Buiatti, Longo, 2013), (Bravi, Longo, 2015), (Calude, Longo, 2015)). 
The notion of "law of nature" in western science has a long and debated history, (Needham, 1951), (Roux, 2009). The understanding of this debate we propose here is that, since Descartes and, more precisely, since Newton, but not before, we could rigorously define the "laws of nature" by a mathematical writing of equations and/or evolution functions in a given space of parameters. This provided the very conditions of possibility for doing physics, by the a priori of space and time, as Kant philosophically framed ${ }^{7}$.

In the XIXth century, besides the Cartesian parameters, observables were explicitly added as a condition for a complete determination. That is, the so called "phase space" was gradually made precise, most prominently by Hamilton, Boltzmann, Poincaré and Gibbs, by adding momentum to position and energy to time. By the end of the century, these pairs turned out to be the crucial "conjugated variables" of non-commutative quantum measurement.

In order to form a phase space by these extensions of space-time, how where the observables chosen ? Both energy and momentum are fundamental invariants of physics: they are characterized by universal conservation laws. And these are understood, in reference to Noether's theorem (see sect. 2), by an even more fundamental mathematical property: symmetry, since they are described by time or space invariance as symmetries in the equations. In the XXth century, the further geometrization of physics, from Einstein to H. Weyl to A. Connes (1994), produced even richer geometric framings of (phase) spaces to make inert matter intelligible.

In biology instead, we claim that the impossibility to give a priori the phase space of evolutionary trajectories does not allow the equational writing of the laws similarly as equations consistently specify the dynamics in physical theories as geodetics, see also (Longo et al., 2012; Longo, Montévil, 2014; Koppl et al., 2015). Our argument here will be based on the role of history, as specified in sect. 1.1, in the determination of the changing phase space of biological processes.

This "negative result" requires an analysis of the positive (constructive) role of history not only in understanding the present states in systems of the living, but also in determining their future dynamics (actually, their evolution spaces). In physics, the mathematical determination allows to frame the future developments, by making predictions (solving equations, computing evolution functions) and/or by assigning probabilities to the different future outcomes, in presence both of classical and of quantum unpredictability. The probabilistic analysis is possible, at least in principle, because the (possibly unpredictable) trajectories will take place in pre-given phase spaces including Schrödinger's trajectory of an amplitude of probability, which unfolds in a Hilbert space. A probability measure is, roughly, the ratio between the expected cases and the set of all possible ones, in the intended space (more formally, Lebesgue measure or other "measure" theories are used). What is unpredictable is a quantity, within a pre-given dimension or observable (the peculiar cases of statistical and condensed matter physics are discussed below). The infinity of the phase space, even its infinite dimensionality, is not a problem: their mathematical symmetries allow to define/compress it axiomatically in finitely many words. This is the further challenge we will mention in biology: the continual symmetry changes in biological dynamics, as spelled out in (Longo, Montévil, 2014; 2015), do not allow to apply a similar a priori formalization of the phase spaces.

We thus deny the possibility of pre-giving a definite phase space in biology (and we dare to extend the analysis to human contexts) and motivate it here by the crucial role of history for the knowledge of the present and the overall dynamics, in the strong sense of the determination of the very phase space. This space of pertinent observables and parameters (phenotypes and ecosystems) continually changes, in ways depending also on past contexts - this is our thesis. Randomness as unpredictability, thus, moves from the impossibility of predicting a quantity within a pre-given phase space, like in existing physical theories (including, with some caution, in statistical physics,

\footnotetext{
${ }^{7}$ The aspects of history and philosophy of science in this analysis are the core issue of the project directed by the author at the IEA of Nantes, "Lois des dieux, des hommes et de la nature" (2014-2020), in collaboration with historians and jurists: http://www.iea-nantes.fr/rtefiles/File/projet-giuseppe-longo-2014.pdf .
} 
see below), to the constitution of the very phase space - it qualitatively affects knowledge. This was understood in reference to "enablement" in (Longo et al., 2012) and in (Longo, Montévil, 2014). In this paper, we stress here the role of past phylogenetic trajectories and of rare events in novelty formation. As a consequence of all these issues, and in contrast to the mathematics for physics, randomness, in biology, at the level of phenotypes, cannot, in general, be associated to a probability measure, as the possibilites, i.e. the list of possible observables and parameters, changes along historical time ${ }^{8}$.

Organisms can "stand" the changing frames, e.g. changing ecosystems, because of their autonomy, in the Varelian sense, (Moreno, Mossio, 2014). Autonomy is not independence from the context: the historicized invariance of the permanent reconstruction of the components that produce the life process, preserves itself through changes, by adapting along ontogenesis and phylogenesis. Moreover, the novel understanding of autonomy as "closure of constraints", within a local spatiotemporality and relative characteristic times, proposed in (Montévil, Mossio, 2015), may provide a link between theories of biological autonomy and the reflections here on history dependence and variability: autonomy adapts by changes, while respecting the characteristic times and the stability of constraints' production and regeneration. That is, a step needed to join the theory in (Montévil, Mossio, 2015) to our historical perspective would be a close analysis of the general historicized invariance proper to "closure of constraints".

Finally, we will stress that even a complete or infinite "instantaneous description" of the present of a system of life, even the one perfectly available to Laplace's Daimon, is formally incomplete for the purposes of its "theoretical determination", in contrast to (classical) theories of the inert: first because of the role we give to history, second because any biological process can only be examined in an extended present, a time interval as given by our notion of extended criticality w. $r$. to the time parameter, see above.

\section{4 - A negative result?}

As we said, the reflection presented here may be a possibly minor, if right, negative result in biology: no way to pre-define the phase space of evolution, because it depends also on partly forgotten histories; no way to assign probabilities to future paths. All or most historians, including of biological evolution, may have already taken for granted these remarks in their work. Our aim here is to frame them in a scientific context and to propose some principles for understanding its relevance.

Observe that often or most of the time, negative results opened new ways to science: Gauss claim that Euclid's fifth axiom could be consistently negated, Poincaré's Three Body theorem (that he called a "negative result"), Gödel's theorem .... The first set the basis for Riemann's differential geometry (and then to Relativity Theory), the second to the modern analysis of dynamical systems (their geometry), the third to Logic as a Mathematical discipline (Computability, Proof and Model Theories were all born after the incompleteness theorem and for good reasons, see (Longo, 2010)). One could add Einstein's derivation of a fundamental property of Quantum Systems, entanglement,

8 The changing phase spaces of biological evolution was explicitly mentioned, in different frames and languages, independently by (Kauffman, 2002) and (Bailly, Longo, 2006). Yet, we were all preceded by R. Thom, in his papers in (Amsterdamski, 1990). For Thom, in scientific analyses, the mathematical phase space pre-exists to the randomness ("noise") affecting the system (p. 70); thus, "it is the lack of the definition [of the virtual possible] that affects - very seriously - the scientific nature of Darwin's Theory of Evolution.” (p. 271). Following Darwin, instead, we work at a science where changes occur at the very level of the phase space, of the virtual possible, in Thom's terminology, and where random events may modify it. Thom's remark resembles the insight in (Einstein et al, 1935): “Quantum Mechanics is incoherent or incomplete, since it implies particles' entanglement”, a property that they formally derive in QM and that contradicts the separability of distinct and measurable events - an absurdity in Einstein's view, as much as Darwin's theory is not scientific for Thom. Great minds may see the key point, even when they are wrong: Darwin's Theory of Evolution does not allow to pre-define the "virtual possible", yet it is scientific; Quantum Mechanics allows to derive entanglement, against Relativity Theory, yet entanglement has been corroborated, it is not absurd (Aspect et al., 1982). 
which is incompatible with Classical and Relativistic frames, see the previous footnote. The myth of the progressive covering of the World by existing techniques and theories, for example of biology by "existing physical theories" (Perutz), is against the very history of mathematics and physics, which have been and are extremely creative areas as for radically new, even contradictory theorizing. Unity of knowledge is a further, difficult conquest as much as the invention of suitable mathematical tools: from Newton and Boltzmann to Weyl and Connes, in the XX century, physical concepts and the search for unifying theories stimulated the invention of brand new mathematics; one should expect the same in biology ${ }^{9}$.

In conclusion, a key and constructive role will be given here to historicity and to the past as such in the determination and the understanding of the present and the future. Thus, our approach crucially differs from existing theories in physics, with the partial exceptions to be mentioned next, which may constitute a possible bridge in understanding these different conceptual frames, as we observed in sect 1.2. Our attempt is based on a methodological transfer (and adaptation) of the key notions of invariants and invariant preserving transformations which are at the interface of mathematics and physics. By this, we try to "objectivize" or propose a scientific approach to rather common intuitions on historicity in systems of life. Hopefully, one day, from this constructive theoretical proposal, our historicized invariants, new mathematical concepts and structures may follow as it happened so often in physics, while this never happened so far and as far as we can say, in the analysis of biological phenomena.

\section{2 - History and path dependence in physics, some comparisons}

In cosmology, the temporal path in the formation of stars, planets and their aggregations, that is their history, is closely analyzed, as cosmology is largely a "historical" science; moreover, many assume today an origin of the arrow of time, which further suggests the relevance of history (the Big-Bang Theory). Yet, the formal determination (by equations, typically) of a cosmological system is given by its current state of affairs, (Islam, 2001). Moreover, up to now, the historicity given by an origin of time is incompatible with Relativity Theory. By Noether's Theorems, energy conservation is understood as a time symmetry in the equations of movement (KosmannSchwarzbach, 2010; Bailly, Longo, 2011; Longo, Montévil, 2014), that is, as a time translation that forbids an origin of time: a first hint concerning the difficulties of having history in physics, which has different responses so far (such as considering time as moving backwards asymptotically towards its “origin”) ${ }^{10}$.

In general, very interesting "history dependent” physico-mathematical systems have been developed following Hertz's original notion of anholonomic system (path dependent systems, see (Berry, 1990) for a survey). The simplest and most paradigmatic case is the mathematical treatment of parallel transport in Levi-Civita's work, which is part of Einstein's use of Riemann's geometry: given points $\mathrm{A}$ and $\mathrm{C}$ and a vector in $\mathrm{A}$ (see the figure below), the orientation of the vector,

\footnotetext{
${ }^{9}$ Also H. Weyl considers the mathematical framing of natural sciences as based on the preliminary construction of a pre-given mathematical space of possibilities, to be a priori set in the background of any analysis. As this must be grounded on fundamental pre-listed symmetries and their possible breaking, he hints to the difficulties this may present as for a mathematical foundations of biology (Weyl, 1949).

${ }^{10}$ Globally, astrophysics is more a science of some fundamental, largely invariant, processes, such as star or planets' formation (Longair, 2006). Yet, the comparatively unbalanced amount of chemical elements is often explained by invoking a particular history of the universe; this is analogous, though, to the understanding of the particular position of a ball downhill by knowledge of its path: an original symmetry breaking or the iteration of a few of them fully describes the state of affairs (see the following examples on "path dependence"). A more interesting example is given by alloys. The precise course of annealing can cause the same alloy to end up with drastically different properties: traces of the past (or "shape memory") play a role in some critical transitions or bifurcations in the intended transformation. However, the entire process and its time are analyzed in a pre-defined phase space. Even a recent radical stress on historicity in cosmology, the changes of value of the fundamental physical constants, refers to changes of numerical values within pre-given dimensions, that of G, c, h (or of a-dimensional constants, such as alpha), see (Uzan, 2011).
} 
transported from A to $\mathrm{C}$ on a sphere (more generally, on a space of non 0 curvature), depends on the path followed by the transformation from A to C (e.g. when along the shortest path or via B), while it is independent from it in Euclidean spaces.

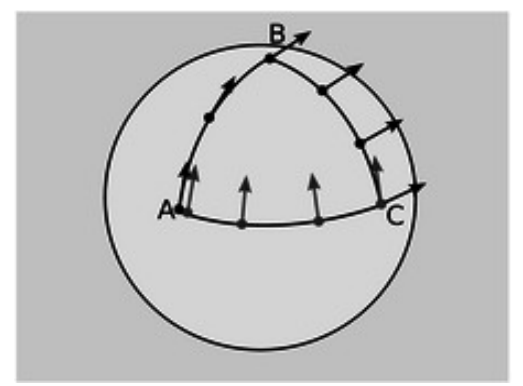

Of course, this is relevant also for the cosmological-relativistic theories above. However, if one adds the oriented vector as a pertinent observable (e. g. as momentum), this Riemannian case gives the general sense of this kind of path dependence: different paths give different results (different "points") in the phase space (the mathematical space of all pertinent parameters and observables), even though they get to the same point in space. Thus, knowledge of the state of affairs in the phase space, including momentum, gives a complete determination of the system, independently of history; more precisely, the traces or consequences of the path followed are fully reflected in the (phase) state, thus, they may be disregarded, as such, for a complete determination of the dynamics.

A more subtle type of history dependence in physics are provided by cascades of transitions through singularities, in Thom's Theory of Catastrophes (Arnold, 1992), or through critical phase transitions in Theory of Criticality (Binney et al., 1992), whose nature and order may matter in the determination of the final result. That is, the presence of a singularity or of a critical point may "deform" a geodetic and lead to a different state. However, in general, knowledge of the paths, when viewed in the pertinent phase space as above, is included in the phase state ${ }^{11}$.

Along these lines, but in a more pertinent way for our purposes, statistical physics and condensed matter physics describe several path dependent phenomena. For example, some extensively studied stochastic processes, such as edge reinforced random walks and vertex reinforced jump processes, where particles tends to come back more often on sites they have already visited in the past, are considered history dependent stochastic processes (Disertori et al., 2015). Yet, on one side, these reinforcements are inserted in the data, thus in an extended phase space, on the other, the randomness of the environment is given as Markov chains, where the probability of the next state depends only on the current state and not on the sequence of events that preceded it, and this within a pre-given list of possibilities.

In other complex cases, whose description goes beyond the scope of this paper, the discussion is open whether the addition of otherwise implicit parameters and observables may allow an interpretation which generalizes the one above on parallel transport and cascades of (critical) transitions. That is, whether history dependence may be understood as different paths producing different results in a suitably extended, yet pre-given phase space. However, the situation seems globally rather well understood similarly as the variable dimensionality in statistical mechanics ${ }^{12}$.

11 This is the case also for equilibrium thermodynamics, where path dependent observables (often called variables) are entropy, enthalpy, pressure ....

12 In statistical mechanics, as recalled in (Longo, Montévil, 2014), one may have a randomly varying number of particles. Thus, the dimension of the state space, stricto sensu, is not pre-defined. However, the range of possibilities is known: the particles have a known nature, that is the relevant observables, the equational determination and the probabilities of each particle's phase space are given. In other terms, even if the number of dimensions of the space may be unknown, it has a known nature and probabilities — we know the probability it will grow by 1, 2 or more dimensions, and, most importantly, they are formally symmetric. Then the number of (possibly extra) particles just becomes a new parameter. Thus, the situation is delicate, but mathematically fully mastered (see (Sethna, 2006), for 
Note finally and more crucially, that far from equilibrium (possibly stationary) structures have no evolutionary history: at most, they follow standard individual evolutions. In our terminology, they have the time of processes, but not an historical time. A flame, a Benard cell, a micelle ... irreversibly go through different states, under a flow of energy or matter, and this process may be timed. However, flames, Benard cells, micelles ... on Earth, are always of the same type, since 4 billions years, and they may be described in a pre-given phase space. This is why we consider them as processes that individually and irreversibly change along time, the time of the process, yet they do not have a historical evolution. This is not the case as for biological organisms and their 4 billions years of a rather rich history, scanned by major changes of the pertinent observables (and parameters) and by rare events, that we will analyze in section 9.

The challenge in biology, this is our claim, is that the very dimensions as well as the pertinent observables and parameters, that is the biological phase space, result also from and depend on a historical path and cannot be pre-given. These changes make history and its time, jointly to the role of rare events. As for now, we stress that, in contrast to the far from equilibrium processes mentioned above (flames etc), which are spontaneous self-organization of flows of energy or matter, organisms do not (spontaneously) self-organize, but use and constrain those flows (Montévil, Mossio, 2015); moreover, they greatly changed the structures of these constraints, from early bacteria to today's organisms. The further difference we add here is that organisms continually use also traces of their history, in particular a fundamental physico-chemical trace and a major inherited constraint, strongly canalizing development: DNA (see below).

We start our analysis by an (apparent) detour on cognition.

\title{
3 - Memory in cognition: an example of historicized invariance
}

\author{
"Imagination ... Its true name is \\ deformation of the memory of sensations" \\ P. Valéry, Cahiers, 1974
}

In order to discuss the role of history in systems of life, it may be useful to start from a specific component of (animal) activity: retention and memory, as fundamental traces of a history. Both preconscious retention and conscious memory have a major functional role in animals and humans: they are needed for action, as they allow protention or expectation ${ }^{13}$. In (Longo, Montévil, 2014), we suggested a simple mathematical representation of biological retention and protention, where protention mathematically depends on retention ${ }^{14}$. This produces a peculiar coefficient of protension, depending on retention in the intended organism, that we called "biological inertia". This is analogous to inertial mass in physics, which, as a coefficient of speed, gives a momentum; biological inertia may express the abstract "weight" (or trace) of the past in protensive action.

Besides the complex issue of path dependence in physics mentioned above, there exist inert materials that have a sort of "memory", e.g. in the case of relaxation phenomena or alloy formation, yet their role for protention, as in biology, is outside the scope of existing physical theories. Instead, some retention of the past seems to be present even in eukaryotic unicellular entities as well as apparent protention (a paramecium, for example, may retain - memorize, previous paths in the protensive search for food (Misslin, 2004)). As a matter of fact, many claim that as soon as there is life there is agency, thus cognition, thus some sort of retention and protention. Yet, we focus on the least brain-body activity as locus for the formation of an active trace of the past, a weak form of

an introduction).

\footnotetext{
13 A classical example of protention is the predator's eye jerk preceding the prey's trajectory, (Berthoz, 2000).

14 There exist relevant neurophysiological and neuro-imaging evidence for (pre-conscious) protention and the dependence of protention on retention (Szpunar et al., 2007, Botzung et al., 2008).
} 
invariant, in multicellular animals. As pointed out in the examples below, retention and protention concern the entire organism in its relation to the environment.

In mathematics, an invariant is fully defined or known when the class of transformations that preserve it, exactly, is fully defined or known ${ }^{15}$. In biology, so far, we can only give an informal, yet mathematically inspired definition of invariance: what is "relatively" preserved under certain transformations of the changing (phase) spaces. It is thus a relational definition: invariance depends on how a mathematical conceptualization is given by or relates to the context, under transformations of the very context (see (Marinucci, 2015) for a synthetic approach to biology as a relational theory). As these transformations happen in time, we may call these invariants, proper to biology, historicized invariants.

We are thus transferring to biology a concept, or, more precisely, a methodology for the formation of a concept. This is invariance under transformations, with the historical variability and flexibility proper to organisms (or cognition) and their theories. The informality is not due to a lack of rigor, but to the different nature of structural stability in biology vs. mathematics and physics, to which we will return below.

Let's now go to our point in cognition, where we first apply our theoretical proposal. In animal cognition, pre-conscious retention (and conscious memory) are forms of construction of invariants for the purposes of action. That is, in order to move, capture a prey, act ..., we the animals do not need to recall exactly nor explicitly the past retained processes, nor all the details of manifold contexts from which they originated as a relatively stable invariant, even not in a distilled, retained form: we learn to precede a trajectory by forgetting the details of the object pursued and the context in early experiences, but retaining what mattered and matters for the action. That is, retention singles out what is crucial for the ongoing activity; it excludes by this the details that are irrelevant for it. Retention (and memory) is selective and forgetful: it forms by this a relatively stable invariant as the result of manifold activities, by negatively selecting and thus forgetting what is irrelevant where "irrelevance" is relative to the ongoing activity and its context. It is retained what matters for action and this allows to iterate the action in changing, yet partly similar environments. Then, the protensive action uses whatever it has been selectively retained and that seems pertinent to the new context by "interpreting" the trace of the past, i.e. by giving meaning to it in a new context ${ }^{16}$. Thus, the retention of an activity and of a context may be considered as the construction of a (biological and cognitive) historical invariant, to be relatively preserved under transformations of the frame.

Protention further stabilizes or modifies the retained invariant, while interpreting it, as reactivation of a trace of the past according to what may be relevant in a new context of action, since recalling itself is a transforming activity. The fixing of a retained action/interaction with the ecosystem and the very evaluation of what is "relevant" for a protensive action, both result from the relational structure and from possible transformations of the internal and external context of an organism, and this for the purpose of facing the future. This is what we mean by saying that animal retention and memory exist for the purposes of action, beginning with the simplest animal movement, the least form of protention (Berthoz, 2000).

In short, a retained invariant is historicized, both as a trace of the past and as a continual reinterpretation of this trace, in a semiotic sense. In our perspective, this may crucially contribute to create novelty: facing uncertainty by using a vaguely similar retained experience produces or forces the invention of a possibly new attitude or response. As we will see for biological evolution in sect. 4.2, novelty is often the result of reorganization of (phenotypic and genetic, in that case) traces of the past. Typically, for future action, one may use and recombine different retained experiences or

15 An elementary example: a straight line may be defined as an axis of rotations, that is as the invariant of a group of symmetries, which are transformations of the tridimensional space in itself.

16 As for the difficult notion of "interpretation” by life forms, we do not mean here conscious "meaning as reference”, but en-action in an "umwelt" in the sense of von Uexküll, (Brentari, 2015), or meaning as encored on action, in a broad, (bio-)semiotic, sense (see also Deacon's reference to Peirce, e.g. in (Deacon, 2015)). This further relates to the rest of the paper, which is on biological evolution. 
(pre-conscious) traces of the past; the use of one or the other, or a blend of them, may depend on minor, un-measurable differences in the present - a form of multi- or bi-furcation due to traces of past histories. Jointly, retention and protention are an interpreting construction and reconstruction of the past.

In summary, the network of past experiences produces a cognitive (retained) invariant, as the result of a sound activity in different frames, as a distilled or interpreted trace of the past. This invariant is further modified when retention is used for protention: the use of memory is never passive, as we recall what is useful for an ongoing action or in the perspective of an action, sometimes radically restructuring the existing mental traces. Memory is always interpreted. Along this line, (Edelman, Tononi, 2000) considers the action of recalling as produced in the brain when it "puts itself in an already lived state", compatibly with its current state. This brain's state is thus the result of a past activity in a possibly no longer existing context, where it had a specific sense, e.g. the "meaning" of a former action; to this, it adds the sense of an ongoing activity. While depending on the way the brain (and body) were structured in the past, this iteration of its state is never identical as it is produced in a changing brain-body relation, in a (new) context and for a (new) purpose: in this sense, it is interpreted. The entire process constitutes a typical example of construction and use of what we call a historicized invariant.

This dependence of future action on the past may be seen as the never identical reconstruction of the previously formed invariant and production of a new one: if the spaces of lived experiences is changing, then also the transformations that define the historicized invariant change. In short, forgetful and continually reconstructed retention or memory are essential components of protention and thus contribute to produce novelty in a changing context: the space of possibilities or phase space of activity continually changes. We stress, of course, that a brain works only in its preferred ecosystem, the head of a living animal, which implies the activity of its entire body ${ }^{17}$. Amazingly, the retention of an activity for future action may be also preserved in the body and later go back to the (regenerated) brain, (Shomrat and Levin, 2013) ${ }^{18}$.

In other words, the partial instability of what is stable, the reconstruction and use of a (relative/historicized) invariant constituted in action, is at the core of biological adaptation, in particular by the most plastic component of an organism: the brain. The transformation towards a new context of a historicized invariant of past actions allows the permanent invention of new solutions when facing the new context, by an opportunistically changing reference to the past, by a re-interpretation of the retained invariant. This may help to produce novelty even when revisiting a familiar context, but on the grounds of a greater or new experience. Indeed, the diversity of the lived experiences contributes, also by their blend, to the varieties of the possible responses to new challenges, thus to the stability or even the survival of an organism. Retained rare events (see section 9) may also and crucially contribute to the cognitive history of an individual, since rare events may affect memory with particular emphasis.

As we are discussing of cognition, we may explicitly say that specific brain and body conformation and deformations have a meaning in a given context, in particular in the context that contributes to shape them. In a changing context, these structures, which may also have changed by the continual (critical) transitions of life trajectories (see below), acquire a new meaning, due to the new relational structure in which they are embedded. And this may go from the individual transformation/invention of new praxes and concepts, to the novelty in collective forms of "being

17 (Prochiantz, 1997) observes that a pianist thinks also with the hands, a dancer with the feet. Neural structures and organs (muscles) are jointly modified and retention is distributed (see also the analysis of vestibular memory in (Berthoz, 2000)).

18 Planarians are shown to recover some memory after their cut head regenerates, as if the bodily traces of a past and memorized activity could influence the newly formed brain (Shomrat and Levin, 2013). As observed in the previous note, it is known that pianists and violinist have visible neural synaptic reinforcement somehow corresponding to local muscles increase (yet, it is not known whether the experiment with planarians would work with pianists or violinists, for lack of volunteers). 
together” and in the world (in economy, for example, (Koppl et al., 2015)).

So far for the construction, transformation and use of historicized invariants of action in a context, in the form of retention and protention, at the core of the dynamics of cognition. We insist that the conceptual transfer we operate from a mathematical methodology must adapt to the biological context. For example, the notion of straight line as an invariant (see the footnote above) is perfectly suitable for the description of inertial movement, in physics, as rectilinear uniform movement. Note that Galileo's inertia is an asymptotic/limit property, never realized in the physical world. Yet, by comprehending from the limit horizon all possible movements, it makes them all intelligible: Galileo could then analyze what affects inertial movement, e.g. gravitation and friction. In the relation of mathematics to biology, this form of abstraction or of limit construction may not work as such, at the level of phenotypes. Thus, we use, so far, conceptual analogies: suitable mathematical tools for these concepts may be the result of further invention, as it continually happened in the history of physics ${ }^{19}$.

We will further discuss memory and cognition, once we have tackled the more properly biological aspects of the role of history in phylogenetic dynamics.

\section{4 - Biological observables and their evolutionary dynamics}

"The entire past of an organism may be reflected in its world",

(Miquel, 2015)

\subsection{Enablement, exaptation and non-optimality in evolution}

Similarly to physics, proper biological theorizing begins by singling out the pertinent observables (and parameters, if possible) ${ }^{20}$. Darwin's theory, for example, focuses on phenotypes, organisms and species. His two fundamental principles, grounded on observation, descent with modification and selection, apply thus to the analysis of inheritance of and within these observables. The choice of the observables depends on "what matters" for the theory, such as conservation properties in physics, as mentioned above, or, in the case of thermodynamics, on the peculiar properties of a novel and very original observable, entropy, which does not need to be conserved. Even when the conserved properties do not change, the unity of the different fields is a further quest, such as in the one century old search for a theory unifying the relativistic and the quantum fields. Similarly, biological theorizing requires its proper frames; the unity with existing or new physical theories must be constructed, as we said: it cannot be an imposed metaphysics, based on the transfer of technical tools as such from one discipline to another ${ }^{21}$.

Mathematics and physics are grounded on a perfectly stable (formal) definition of invariance

19 The co-costitutive history of physics and mathematics, the latter being a limit construction, is grounded, in particular, on the genericity both of mathematical concepts and of physical objects: they are invariant of the theory and experiments, e.g. any right triangle works for a fully general proof of Pythagora's Theorem, any stone or electron would do in a pertinent experiment, from Galileo to Planck. In other words, they are symmetric or invariant under permutations with other right triangles, stones or electrons. Moreover, physical trajectories are specific, that is, they are geodetics in suitable phase spaces. These issues are discussed at length in (Bailly, Longo, 2011) and (Longo, Montévil, 2014). In our approach, the mathematical tools for physics cannot be transferred as such to biology, except for some local applications (some aspects of morphogenesis, for example), since biological objects (the organisms) are specific (historical, individuated) and their trajectories are generic (they are possible evolutionary and ontogenetic paths), a crucial duality with physical theories, see (Longo, Montévil, 2014).

20 Galileo started the dance of modern physics by observing momentum: inertial movement is momentum conservation.

21 This is explained at length in the introduction to (Longo, Montévil, 2014). For example, Boltzmann asymptotic unification of classical trajectories and conservation laws with thermodynamics is discussed as a counterexample to prevailing reductionists modes. Boltzmann's approach lead to a new unifying theory and its mathematics: statistical mechanics, see (Chibbaro et al., 2014) for this and more on the anti-reductionist history of physics. 
since their objects are generic. In biology instead, the structural stability of an organisms, but of an organ or a population as well, is due to the organisms' closure or autonomy but also to variability, which allows sensitivity to the context, adaptation and diversity and is based on the specificity (thus historicity) of organs and of individuals; a population or an organ is more stable (by resilience or fault tolerance, typically) if diverse, and this is so also with small numbers (Bravi, Longo, 2015). These features of life, the role of diversity or lack of (perfect) invariance of individuals in particular, force to understand biological robustness and resilience as different from the notions used in physics, see (Lesne, 2008). In particular, physical or computational redundancy are very different from biological diversity (and also from Edelman's notion of degeneracy, see below). Diversity is the result of historicized invariance, as the specificity of each organism depends on its phylo- and ontogenetic history. As a matter of fact, as well known in biology laboratories, observations and experimental activities always require the best possible knowledge of the history of the specific animal or even cell used as a model.

For the purposes of our analysis, thus, we consider Darwin's observables and their historical dynamics. Our aim elsewhere is to unify phylogenesis with ontogenesis, partly in an EvoDevo perspective (ideas in this direction are proposed in (Longo et al, 2015) and further developed in (Soto, Longo, 2016)). In this frame, inheritance is a crucial object of study. Our aim here is to analyze how knowledge of history as such, not just by its current consequences, must be taken into account in any attempt both to "determine" the present and to analyze the future evolution of an organism. That is, we claim that life systems cannot be considered as "state determined systems", as knowledge of the historical path toward the current state plays a direct role in the theoretical determination of present and future dynamics.

Whatever a proposal for a biological determination may be, it should clearly yield unpredictable dynamics, as only fools claim to be able to predict, by solving equations and computing, or by assign probabilities, the list of phenotypes in one million years, a reasonable evolutionary time scale $^{22}$. Yet, some claim that this is just a matter of a deterministic unpredictability, in the sense of non-linear dynamics and, thus, analyze evolutionary paths as geodetics in pre-given phase spaces, possibly under the assumption that genes contain the complete determination of phenotypes (see the optimization in population genetics in the approach by (Grafen, 2014), where reproductive maximization drives evolution, following the Modern Synthesis, (Huxley, 1943)).

The least critique one may address to this gene-centered approach, largely based on classical dynamics, is a reference to quantum indetermination which seems present at the molecular level, both in genetic and epigenetic phenomena (Arndt et al., 2009), (Buiatti, Longo, 2013). In short, while physical randomness is unpredictability, either classical, thus deterministic, or quantummechanical, thus due to intrinsic indetermination, biological randomness is at least a blend and a superposition of the two. A mutation, a jumping transposon ... may depend on molecular events as well as on an epigenetic effect, such as (de-)methylation: quantum and classical randomness may then superpose, in a cell, and, independently or jointly, have phenotypic consequences (see (Buiatti, Longo, 2013) for a survey and references; (Plankar et al., 2011) for relevant aspects of quantum coherence in cells). Thus, a quantum event may engender a variation in the biological observables, the phenotypes, which is at a very different phenomenal level from the microphysical one with its peculiar form of randomness. In particular, a phenotype may causally depend on an a-causal event, in the standard interpretation of the quantum formalism, well beyond then classical geodetics.

Moreover, the interactions of different levels of organization may produce what is called "bioresonance" in (Buiatti, Longo, 2013), in analogy to the Poincaré's planetary resonance that originated the analysis of deterministic unpredictability ${ }^{23}$. This allows to take into account also

22 Independently of life, it is even difficult to assign probability values to the Earth or the Planets positions in the Solar system at that or at a slightly larger time scale, (Laskar, 1994). Yet, the space of possible positions is known: the phase space of the classical dynamics of the Solar System. Thus assigning probabilities is legitimate, though technically difficult.

23 Technically, planets are in resonance when they are aligned with the Sun. This produces major instabilities in their 
epigenetic and environmental interactions and inheritance that are not DNA based (Jablonka, Lamb, 1998; West-Eberhard, 2003; Rando et al., 2007; Nowacki, Landweber, 2009). Then, biological selection applies by excluding the incompatible organisms and by enabling the compatible ones in a co-constructed ecosystem, (Longo et al, 2012), (Longo, Montévil, 2014).

This is clearly very different from engendering a geodetic in a pre-given phase space, an ecosystem to-be, whose possible life forms are completely encoded in the DNA, following the myth of the DNA coded animalcule of the Modern Synthesis. To see the difference, note that a river or dice go along a, possibly unpredictable, geodetic, a specific path in a pre-given phase space and never go wrong. In evolution (but also in ontogenesis), organisms (and species) go wrong most of the time (99\% of species disappeared): most are excluded by selection, a few are enabled. Life generates new life by exploring generic paths (i.e. possible paths, relatively invariant under suitable transformations, not uniquely determined), under internal, organismal, or ecosystemic canalization or enablement, and very often it fails. The result is far from optimal, even less an optimum, at least because there is no pre-given partial order, where a largest element could be defined: the ecosystem is co-constituted by the dynamics and no pre-given choice of (mostly incomparable) observables may allow to fix a partially ordered space with a maximum or even local maxima. Indeed, if an organ or an organism were an optimum in a given phase space, and this optimality were a (essential) component of its fitness, as claimed by the Modern Synthesis, it would be very soon dead, as the ecosystem changes. In Jacob's metaphore of "bricolage" (Jacob, 1981), evolution may use the chassis of an old chair to construct the box for a radio. This box is surely not optimal, but ... one may sit on it, while listening to the radio, a convenient exaptation (ex-post adaptation), in Gould's terms (see the examples of hears, feathers and the many "spandrels" of various sorts ... (Gould, Vrba, 1982; Gould, 1989; 2002)).

The exaptation of inherited traces of the past, jointly to organisms' autonomy, in the sense of (Moreno, Mossio, 2014), contributes to life relative stability by the continual invention of new compatible "solutions", under the co-constructed internal and external constraints of viability. It should be noted that exaptation and bricolage are better understood by departing from the myth of optimality in evolution. Jacob's metaphorical example of the re-use of an old chair to make a nonoptimal radio box, its possible multiple use (also for sitting when listening), is a form of functional "overloading" of organs that is very common in biology: our hands and our brain are the most typical examples - they have been and are continually overloaded by new functions. Primates' hands are good for grasping tree branches, but are not optimal (the thumb does not "oppose" perfectly well), yet, and because of this, they may also be used to caress or to tap or flatten an object with the open hand. In other words, if an organ were designed to be "optimal" in one activity, it would be hard or impossible to use it for different purposes, by a synchronic use or by evolutionary exaptation as a form of biological invention. Optimality for one purpose cannot be such for other uses, thus it would exclude alternative functions, by principle. As we said above, if fitness is optimality in a given phase space, the optimal organ (and the organism) would not stand ecosystemic changes. Moreover, by the relational structure of biological dynamics, it is impossible to isolate one observable, define a partial order with a maximum and attribute this value to a measurable biological function ${ }^{24}$.

orbits, very well expressed by non-linearity, and massively contributes to chaos, even in the Solar System (Laskar, 1994). Yet, this happens within one level of determination, the equations for the planetary system. Bio-resonance, instead, refers to the interferences, by regulation and integration, between different levels of organization in an organism, possibly given by different forms of (mathematical) determination (e. g. statistical networks as for cells' interactions and classical non-linear dynamics as for organogenesis, (Buiatti, Longo, 2013)).

24 This flat application of classical geodetics to phylogenetic trajectories is nicely criticized also by physicists working in biology, see (Goldenfeld, Woese, 2011), which "highlights the importance of collective interactions and the interplay between environmental fluctuations and evolution, which are neglected in the Modern Synthesis” as well as in geodetic/optimality approaches to evolution based on it. The best is to quote their text: “ ... horizontal gene transfer is now known to be present in multicellular Eukaryotes as well, as a result of genome-wide surveys 


\subsection{On biological “creativity".}

It should be clear that we are discussing the various notion of "creativity", "inventiveness" ... as theoretical notions, as they are a form of unpredictability in the intended historical theory, as spelled out in the footnotes above. Thus, we do not impose any metaphysical absolute to the reader: whether or not a, possibly infinitary, "Laplace's daimon" or "hidden variables theory" will one day fully interpret these notions in causal, pre-given Cartesian frames, it is possible, but it is not an issue here. So far, we interpret words such as "creativity" or alike, often used in analyses of life, but unsuitable in existing physical theories, as the appearance of new observables (phenotypes), grounded also on the contextual re-interpretation of past histories. Traces of the past contribute to the construction of new observables, thus of new spaces of ecosystemic interactions, or phase spaces. As summarized in (West-Eberhard, 2005), "the origin of species differences, and of novel phenotypes in general, involves the reorganization of ancestral phenotypes (developmental recombination) followed by the genetic accommodation of change”. For example, the leaf forms of Monstera dubia "have been developmentally duplicated, deleted, and recombined in a multitude of ways during the evolution of the genus Monstera, giving rise to a variety of species-specific ontogenies"; another kind of developmental recombination is "cross-sexual transfer, or the transfer of trait expression from one sex to the other”, see (West-Eberhard, 2005). Also heterochrony (altered timing in ontogeny) in the expression of adaptive traits that previously evolved may contribute to speciation. For example, the three-spined stickleback (Gasterosteus aculeatus) presents both a "limnetic" and a "bentic" form. Their "ancestral population occupies both of the habitats observed in the descendent species pairs and exhibits both phenotypes at different times during its life cycle, a pattern that suggests that the different recurrent forms may have originated not by parallel evolution but by altered timing (heterochrony)" in the expression of those traits (West-Eberhard, 2005). More generally, organisms contextually "interpret" phenotypic traces of the past and re-use them: "Phylogenetically separate, recurrent phenotypes show that a common type of developmental recombination is the reexpression of phenotypes that have been lost because of developmental deletion, alteration of a regulatory mechanism, without extensive alteration of other aspects of the developmental capacity to produce the lost form. ... Very large body of evidence ... shows that phenotypic novelty is largely reorganizational rather than a product of innovative genes" (West-Eberhard, 2005).

Evolutionary novelties may be enabled both by ecosystemic and by genetic changes with phenotypic effects. Their unpredictability radically differ from physical unpredictability: there is no "creativity" in the result of coin flipping or of an electron spin up/down, where all possible outcome are pre-given. Even the new symmetries in quantum or classical coherence structures, e.g. in the so called "emergence" of (self-)organized structures in critical transitions or (self-)organization of flows in far from equilibrium dynamics, do not create novelty in the biological sense. In these cases, symmetry changes give new physical objects which may be quantitatively different within pregiven or predictable dimensions and observables; moreover, these objects spontaneously emerge, in contrast to biological organisms, and are always similar: they at most depend on a processual time, along always similar paths, as mentioned above. Instead, phase space changes, in biology, scan the

published in the past year or so ... Inheritance of acquired characters ... not only through horizontal gene transfer (e.g., in microbes), but also through so-called epigenetic mechanisms that bypass the usual modes of inheritance, especially in ciliates. Not only is the Modern Synthesis afflicted by strong interactions, but its very foundation is questionable. The evident tautology embodied by "survival of the fittest" serves to highlight the backward-looking character of the fitness landscape: Not only is it unmeasurable a priori, but it carries with it no means of expressing the growth of open-ended complexity and the generation of genetic novelty. Thus, the Modern Synthesis is, at best, a partial representation of population genetics; but, this on its own is a limited subset of the evolutionary process itself, and arguably the least interesting one” (page 383). The "a priori unmeasurability” for us is due on the coconstitution of phylogenetic trajectories and their phase space; the open-ended random complexification of organisms is formalized in (Bailly, Longo, 2009), (Longo, Montévil, 2014). 
historical time and contribute to biological novelty.

\section{5 - Towards the future: knowledge and unpredictability}

As we mentioned, if phenotypes are the pertinent observables, since a (possibly a-causal) quantum event may modify a phenotype (see (Buiatti, Longo, 2013) for a survey), this is enough to derive the intrinsic unpredictability, in the quantum-mechanical sense, of the changes of the very phase space of biological trajectories. Yet, this is not our main argument here. In general, selection and enablement apply at the organismal level, on the grounds of phenotypes: variations continually propose what will be eliminated or enabled by and in a co-constitued environment. This coconstitution does not exclude downwards guidance of variations by the ecosystem, as we said ${ }^{25}$, typically by the control of DNA expression, by (de-)methylation or even by changing physical constraints on the chromatine or on the nucleus membrane (Bécavin et al, 2012), (Desprat et al, 2008), (Fernandez-Sanchez et al, 2010 ).

However, our aim here is mainly the investigation of the role of history for the understanding of the present, thus, a fortiori, for any analysis of future biological dynamics, that is for the analysis of (un-)predictability. If this role is relevant, our proposal may contribute to a new knowledge frame and would radically depart biological analyses from existing physical theories, as "state or process determined theories" - which does not forbid a search for future unity, that is the invention of a new, unifying theory and mathematics, possibly by references to "possibly bridging theories" (criticality, statistical or condensed matter physics ...). As we already stressed, physicists have always been searching for theoretical unity and new mathematics, along history, not "reductions", from Newton's unification of Galileo's falling apples and Kepler's planetary orbits to Boltzmann's or Maxwell's work, the latter relating electromagnetism and electric fields, to today's search for unification of astro- and micro-physics, see (Chibbaro et al., 2014).

\section{6 - Invariant traces of a history}

Evolutionary inheritance is a many dimensional phenomenon (Jablonka, Lamb, 2008). In particular, traces of history are present at the genetic level, but also in proteomes, membranes etc... up to cultural inheritance. It is well-known that embryos inherits RNA molecules from the mother's cells surrounding the zygote; the immune system as well is initially inherited from the mother and provides an example of inheritance of an acquired character (Lemke, 2004).

In (Longo, Montévil, 2014), phylogenetic and ontogenetic paths are seen as the permanent reconstruction, by changes of theoretical symmetries, of a coherence structure: the organismal unity with its internal and external correlations. Its viability, as internal and ecosystemic coherence, is maintained while changing and by changing: adaptation and exaptation, as organs' adaptation from past functions, are essential to survival. This peculiar dynamic behavior of the living yields extended critical transitions, in the sense above, as it extends the notion of critical phase transition in physics to an interval of all pertinent parameters (time among others). In the mathematics of physical criticality, this notion is described as a transition on a mathematical point ${ }^{26}$. At the pointwise critical transition, a coherence structure is produced or changed, by symmetry changes.

In biology, in our view, one can make no sense of phenomena on a point of time, nor of other parameters. The instantaneous picture of a falling stone, of a snow-flake ... gives an informative image of the inert object; a one instant dynamics of an organism may only render the anatomy of the dead animal: functions and biological autonomy are totally lost. Any proper biological understanding requires some time interval of analysis. And, within this interval, the least cell reproduction is the reconstruction of a new coherence structure with new symmetries: the new cells

\footnotetext{
${ }^{25}$ See the footnote above on (Goldenfeld, Woese, 2011) and (West-Eberhard, 2003) for more extensive references.

26 This is needed for the application of the renormalization methods to this phenomena, (Binney et al., 1992).
} 
are similar but not identical (slightly different DNA reconstructions and bi-partition of the proteome, of the membrane ... a symmetry breaking). In a multicellular organism, the tissue matrix and tensegrity structure, the intercellular correlations are reconstructed, but never identically. These symmetry changes characterize, in our perspective, all life trajectories and are at the core of variability, thus adaptation and diversity, as crucial contributions to biological stability (Longo, Montévil, 2014).

Yet, something (a lot) is conserved and this is inheritance, both at the cellular and the multicellular level. The changing, yet the inherited organismal structure in the transformations of the ecosystem include at least DNA, RNA, proteome, and membranes: in our terminology, they are historicized (material) invariants. By a network of interactions, within an inherited ecosystem, ontogenesis yields the tissue structure and organs' autopoietic correlations, i. e. the global functional unity or the coherence and autonomy of the organism, as a coherent unity of dynamical constraints, in the sense of (Montévil, Mossio, 2015). That is, the historicized invariant traces of the past are the result of and produce a permanent reconstruction of relatively stable, thus locally invariant structures at all levels of organization. Note that we superpose descriptively ontogenesis and phylogenesis, since we consider an organism's phylogenetic trajectory as the sum of ontogenetic paths, subject to the same principles (Longo et al., 2015) ${ }^{27}$.

\section{7 - Constructive relational spaces and invariance}

In spite of the non-chromosome-centered nature of this analysis, let's start with a reference to DNA, a fundamental chemical trace of the evolutionary history of an organism. This trace is permanently used by each cell, during ontogenesis. Similarly as for any component of life, DNA functions may be understood only in a relational way: it works and we can make biological sense of it, only in relation to a context, a cell living in a viable ecosystem, including an organism. That is, DNA biological activity may only be defined in relation to its context, as an identical DNA may express the proteins needed, say, in a caterpillar or in a butterfly: an induced metabolism may deeply modify the expression of the same DNA in many social animals (bees, ants ...) etc. In no meaningful sense the DNA contains the complete determination of an organism: in the terminology of (Montévil, Mossio, 2015), DNA is a (fundamental!) constraint of phyo-ontogenesis - the cell, in changing contexts, produces proteins by using DNA as a constraining template, in different ways.

As a matter of fact, the chemical structure of DNA itself is the result of its historical relation to several contexts of activity; that is, it is the relatively stable, historicized, invariant that has been formed and that maintained itself through varying contexts, through time. Mutations, transposons, insertions, by retroviruses for example, and many other forms of horizontal contamination, which we mentioned, may be spontaneous, and due the various forms of random effects recalled above, and/or may be induced by the ecosystemic interactions ${ }^{28}$. When phenotypic consequences of DNA changes are enabled by the context, they contribute to the relative stability of the phylogenetic history of an organism. The stability is relative, as the context may use differently the same or approximately the same molecular as well as any other biological structure, typically by Gould's exaptation or Jacob's bricolage (Gould, Vrba, 1982). For example, the re-use for new biological functions of transposable elements of DNA is a form of exaptation of transcription factor binding sites (promoters and enhancers), previously playing a different role, and it extensively affected the evolution of gene regulation (Souza et al., 2013). Similarly, feathers, from heat regulators, were exapted for display and bird flight. Traces of history are re-interpreted and acquire a different biological meaning in new contexts and are, thus, modified.

In summary, the cell and the organism may use in different ways a given fragment of DNA or the

${ }_{27}$ For a comprehensive view in this perspective, see the journal special issue (Soto, Longo, 2016).

28 In the immense literature on mutations as well as on different uses of DNA in evolution, some recent striking advances are in (Harms M., Thornton, 2014). 
expression of different fragments of DNA. Methylation, demethylation and alternative splicing, among others, are changing forms of random or controlled use of DNA along ontogenesis and evolution: minor (stochastic or oriented) fluctuations in the fragment involved or in the way it is used may give different ontogenetic or phylogenetic paths. Cryptic genetic variation is a further example of this phenomenon. According to (Paaby, Rockmann, 2014): "Cryptic genetic variation (CGV) is invisible under normal conditions, but it can fuel evolution when circumstances change. In theory, CGV can represent a massive cache of adaptive potential or a pool of deleterious alleles that are in need of constant suppression”. Similarly, the increasingly stressed relevance of horizontal gene transfer in evolution (Keeling, Palmer, 2008), requires some knowledge of the past role of the transferred gene in order to fully understand its present and possible future functionalities. Like in cognition, measurable differences in the present or future use may depend on traces that differ below measurement or on different uses of the same trace of a past activity - a form of bifurcation due to past history. The difference with physics should be clear: in physics, the choice of a bifurcation depends on local fluctuations at the "time" or "place" of bifurcation, as path dependence may be taken into account by a suitable extension of the phase space; in biology, it may depend on past undetectable or currently irrelevant variations, which does not exclude that they may have been relevant in the past. The point is that the phenotypic consequences of a fragment of DNA may be understood only in relation to an epigenetic, organismal, ecosystemic context. Thus, as for knowledge and prediction, possibly unknowable contexts in the past, which co-constructed organisms and their DNA, may be needed to understand the manifold possible uses of a fragment of DNA.

As for DNA, thus, its very structure and its biological role are the result of contexts of interactions which span its history. Then DNA maintains a structure and expresses itself in a relatively invariant way as long as the transformations of contexts reproduce similar interactions and enabling frames. Yet, in a changing context, it may be used differently. The same may hold for any other component of an organism. In general, in our perspective, which is a conceptual one inspired by mathematics, these contexts' transformations produce and define the historicized invariance of biological structures: they are needed for the knowledge of the present and in the analysis of predictability. As we mentioned, feathers in dinosaurs were a possible and relatively stable result of an ecosystemic interaction, where, allegedly, thermal exchange played a major role: they lasted for tenths of millions years. Then, only the clade of feathery and flying dinosaurs has survived, a fact that it would have been hard to predict for the early observers of running dinosaurs with scattered feathers; the exaptation of feathers, with changes, has been crucial to their survival. Moreover, the increasing diversity within that clade contributed to its evolutionary stability.

In biology, invariance with respect to ecosystemic transformations is not exact, nor permanent, and its historicized form is essential to organisms' and species' relative, historical stability, by variability, adaptation and diversity. These properties, in a population, in a species ... are an essential component of survival and, thus, of long term stability.

\section{8 - Knowledge of the present and invention of the future}

Following our analysis, in mathematics, objects are defined by their invariant properties, thus in a relational way, by transformations within or between spaces. The conceptual and constructive nature of mathematical structures allows to define them exactly by a pertinent set of transformations within a given space (or Category) or, possibly among different well defined and restricted contexts (different Categories, in the language of contemporary mathematics ${ }^{29}$ ). In an organism, instead,

29 In todays' mathematics, Grothendieck's approach is the finest existing form of relational mathematics and its application to contemporary physics is being organized in this style, see (Zalamea, 2012). The definition of new concepts and structures, in Grothendieck's relational approach, develops but goes beyond the approach based on invariants and transformations, which range from Klein to Weyl and MacLane (the founder of Category Theory): definitions a la Grothendieck are given in the "purest" yet meaningful mathematical frame, so that their invariance 
almost everything is related to almost everything else, and, as we know since Darwin, variations take always place and are always "correlated". This is so also in an ecosystem, though more weakly ${ }^{30}$.

\section{1 - The understanding of present life}

Can we completely understand and determine the structure and the function of a component of an organism, of an ecosystem, by the current (chemical, say) structure and the biological correlations? By continuing the argument above, this is impossible, since each component is the result not only of the current relations, but also of a history of past relations that contributed to produce the existing structures and their functions. We presented DNA as a typical example of this. No isolated segment has a definite function: polymorphism and pleiotropy (one gene related to multiple phenotypes and, conversely, many genes - one phenotype) allow very different functions, beginning with the genesproteins many to many relations - a form of "degeneracy”, in the sense of (Edelman, Gally, 2001).

Degeneracy is present everywhere in biology, from the DNA to the brain. It may be specified as (i) "systemic" degeneracy: a same system contributing to distinct functions; and (iii) "functional" degeneracy: non-isomorphic systems that yield or participate to the same function, see (Bailly, Longo, 2011) for this distinction. It differs from redundancy in artificial devices, computers for example, as the latter is achieved by iteration of identical components (redundancy).

Systemic degeneracy, thus, corresponds to different functions that a same life component may have or may have had along its constitutive history, in different relational contexts. Homologies are consequences of this: a common origin then diverging functions and, possibly, anatomy (e.g. front legs vs. wings in tetrapods) ${ }^{31}$. In some cases, homologies at the bio-chemical level, for example the common "ligand-receptor interactions and their intermediate downstream signaling partners", may show "the functional homologies between such seemingly disparate structures and functions as the lung alveolus and kidney glomerulus, the skin and brain, and the skin and lung” (Torday, 2015). Conversely a given function, may be the result of different evolutionary paths, which allow to understand the different anatomy (functional degeneracy); analogies are typical examples (e.g. insects' vs. birds' wings). The understanding (the theoretical determination) of these phenotypes then depends on the (reconstructed) knowledge of past common roots, possibly enriched by paleontological evidence.

Now, the potentialities of degeneracy are the consequences of a past constitutive history, but do not need to manifest themselves in a given state of affairs. In other words, each component of an organism or an ecosystem is the result of ever changing relational structures that possibly left in that component some traces of the changes at each relevant historical passage. It is thus the biological, historicized invariant resulting from the historical transformations of the context. So, even a complete knowledge, if ever possible, of the present state of the correlations, in an organism and an ecosystem, does not yield a complete determination of the state of affairs nor of its future dynamics, as position and momentum do in classical dynamics: the organism's entire history contributed to the construction of the present, by the past dynamics of the relations, whose possibly lost meaning contributes to the current determination and to the construction of future new meanings, as contextual uses. Thus, in contrast to physics, no pre-given $a$-historical phase space may include all past and future observables and their possible qualitative descriptions, also because past ones may be lost to knowledge or inaccessible to measurement. This does not make Darwin's evolution

and the intended transformations are "intrinsic" to the new notion, see (Longo, 2015).

30 An organism is an ecosystem, with crucial phenomena of symbiosis of different species. Yet, an ecosystem is not an organism: its coherence structure is more weakly correlated, at least by the metrics (but also by the functions). For example, two cells in the same tissue will never depart too far and maintain their organismal functions, while, even within a colony, say, two ... workers ants may go from 0 to tenths of meters apart and still be functional. But, of course, there is more than this as for the greater coherence and autonomy of an organism (Moreno, Mossio, 2014), (Montévil, Mossio, 2014).

31 See http://www.britannica.com/EBchecked/topic/270557/homology 
unscientific, as Réné Thom claims and many try to fix by idealistically forcing the mathematics of physics on top of biology: it is instead at the core of the science of phylogenesis and ontogenesis.

In summary, the biological significance of a component of an organism, from DNA to an organ, has a changing history. The chemical structure of (a segment of) DNA, say, is the result of that history, including the manifold contexts that contributed to shape it and to shape its function, by variation, selection and plasticity. Similarly, only by an analysis of the interplay of homologies and analogies or convergent evolutions of, say, the pulp's and vertebrates' eyes may help to understand their structure and function, as the characteristic of eyes in bilaterian animals is not only due to current functional constraints but are also a consequence of their evolutionary history ${ }^{32}$. Past relational contexts may be difficult to measure or lost, yet their consequences in existing structures may contribute to an understanding of current as well as future possible functions. In general, then, the description in terms only of current functions and relations is necessarily incomplete, because their biological sense is a matter of a historical construction. Moreover, in our approach, it must always be given in an extended present, an extended interval of criticality.

Of course, this adds on top the incompleteness of the mathematical descriptions of exact (pointwise, say) states in physics. As we mentioned, classical measurement is always approximated, it is an interval, by principle (by at least the thermal and gravitational fluctuations) and, in case of nonlinearity of the mathematical determination, this yields classical randomness as deterministic unpredictability. Analogously, though differently, quantum physics integrates, in the theory, probabilities and the indetermination of measurement. In either case, physical determinations, by measurement and mathematical formalization, are synchronic: they disregard past histories - with the peculiar partial exceptions mentioned in the introduction and in sect. 2, still reducible to a phase state analysis in a sufficiently rich phase space or, at most, by integrating in the analysis a processual time. Thus, incomplete knowledge and determination, in mathematical physics, only depend, in principle, on the way we access to current states of affairs, that is on its measurement, up to some attention to the past, in a given phase space, that we mentioned in section 2.

Thus, our argument extends the synchronic incompleteness of knowledge proper to physics, which applies, of course, also to biology, by a diachronic incompleteness of the biological determination. Moreover, most of the evolutionary history is definitively lost, jointly to past genetic, epigenetic or other forms of interactions: we have no more access to them. In physics, by principle, one cannot go below the best classical measurement interval nor below Planck's h for conjugated quantum variables. In biology, the are also inevitable gaps in knowledge and approximations in measurement of past histories.

\section{2 - Inventing the future}

“... no hay camino, ... se hace camino al andar” Antonio Machado

On the grounds of the previous argument, one cannot provide a complete list of the ways a structure where degeneracy applies may actually function in a new context, as this would require also a complete knowledge of the past uses and functions: some may be also reactivated in a new context, though differently, of course. This may recall what we said above on protention based on retention, in animals: a form of ever changing reactivation of a reinterpreted past.

In other words, on one side the entire relational history of an organism or an ecosystem contributes to its actual determination, on the other, it also contributes to the way it may react to an internal or external change: knowledge of future reactions may require at least knowledge of past

32 Typically, in octopuses, the nerve fibers pass behind the retina, while, in vertebrates, they route before it and disrupt it by a "blind spot". The different phylo- and onto-genetic histories of the two neural systems help to (partially) understand this difference and show that there is no optimality, since the vertebrates' eye may be considered '"better" as for the presence of a cornea, but not as for the nerves' routing (the rumor has it that Helmholtz, an early profound analyst of the eye structure, proposed to fire the Designer of the vertebrates' absurdly connected eye ...). 
functions and relations that may be partly or largely unaccessible to knowledge.

We are thus extending physical theorizing by properly biological remarks. If history crucially contributes to the determination of biological systems, it also renders them intrinsically unpredictable, on top of physical unpredictability, by the incompleteness of knowledge, thus of measurement, of the past. Can one give intrinsic limits to these measurements?

In classical physics it is possible, locally, to evaluate the thermal and gravitational fluctuations, thus set a lower bound to the measurement interval (Rowan, 2005). Plank's h seems to be a very robust, though approximated, lower value for measurement (of conjugated variables). In biology, for a fixed observable, the size of the best interval of theoretical approximation in historical measurement of a given observable, if ever possible, should increase backwards with time, in principle. Yet, of which observables and functions is it a measurement, exactly? We may have even lost knowledge of some past "observables" or functions.

Of course, one may interpret this role of an (unknown) past in constructing the future in a positive mood. By re-using invariant traces of a no longer existing relational context, evolution (and ontogenesis) creates unexpected novelty. The re-use is never identical: identical evolutionary, but even ontogenetic paths and contexts do not exist. The role of rare events, discussed in the next section, is sufficient to prove it. Thus, to the reasons from physics for novelty in biology (e.g. random molecular events with phenotypic consequences and bio-resonance in (Buiatti, Longo, 2012)), we added here the role of history in knowledge and determination both of the present and of the future biological dynamics.

The major difference w. r. to existing physical theories, but a partial bridge towards statistical and condensed matter physics, is the ever changing phase space: the pertinent observables, the phenotypes, are the changing structures. It is the very phase space, in biology, that is unpredictable. The mathematical challenge is to move an analysis of randomness from within a given phase space, like in classical and in quantum physics, to the very constitution of the phase space: this yields the impossibility to measure randomness by probabilities (an issue, though, which is very delicate also in physics, see (Longo, Mugur-Schachter, 2014)). However, mathematics is a major human creation; fortunately, it is not already there, in the pre-given Newtonian Absolute Universe of Set Theory: we invented all what was required, so far, for physics and much more. In some cases, decades where needed to frame coherently new physico-mathematical ideas: e.g. Dirac's delta in Schwartz's distributions, (Schwartz, 1951); the mathematical status of Feynmann's integral is still not very clear: so far, it is a "solution without equations", a remarkable invention. As hinted in (Longo, 2015), biology deserves to interact in a co-constitutive way with contemporary mathematics, not just the mathematics created jointly to XIX century physics.

We tried to describe the creativity of life from life, in terms of an intrinsic unpredictability of the phase space (in a sense, the ecosystem), also in view of role of history in biology. An understanding of this creativity in terms of incompressibility of the description of the phenotypes may relate our informal approach to the mathematics of Algorithmic Information Theory, AIT (Calude, 2002), at the purely epistemic level though: the linguistic description of changing phenotypes may be impossible before they happen, it may be thus considered to be time-incompressible - there is no way to generate this description it in advance. This must remain epistemic and just expressed in terms of "wording", as the discrete computational frame of AIT is strongly incomplete w. r. to physical dynamics, that we mostly understand in continua, thus a fortiori for biological evolution. Recall that, mathematically, a structure is discrete, when the "natural topology" is discrete, that is, when the access to data or measurement is exact. This makes rarely sense in physics, even less in biology: complex physical (and biological) dynamics are always dependent on fluctuations and variations that we better understand in continuous topologies and metrics. Randomness as relative unpredictability, at the core of our approach, makes only sense under classical approximated or quantum undetermined measurement. Thus, the incompressibility of phenotypes' description may only refer to our contingent forms of knowledge, and has little scientific objectivity - yet it may 
provide a hint to the difficulties.

By these remarks, we added the issue of approximated historical or diachronic knowledge and measurement to the problems of synchronic measurement. And we moved randomness as unpredictability, from within, to the very construction of phase spaces: what is unpredictable is not the numerical value of a given observable, but the space of observables, a qualitative judgment on nature.

\section{9 - The role of diversity and rare events}

We already extensively discussed diversity as a component of the structural stability of life, in species, populations and organisms. Diversity also depends on randomness, which is far from being noise, in biology, as stressed in (Bravi, Longo, 2015). In that paper, we justified the constructive role of diversity also for organismal stability, and exemplified this by several references (immune system, lungs, liver $\left.{ }^{33} \ldots\right)$.

Diversity is the result of the specificity of each individual cell and organism: for example, even individual liver cells are the result of a (short) history, which is sufficient to generate diversity by a cascade of symmetry breaking (in this case, by DNA copying and proteome's partition at cell reproduction). By this, each cell and, more generally, each biological individual, is rare, as specific/historical. Yet, this diversity, as individuality or specificity within a range of viability, contributes to the global stability and viability of an organism, a population, a species, in the way hinted in the examples above. Typically, in a species, diversity may be compatible with sexual reproduction, whenever one considers this criteria, inter-reproducibility, as a (weak but) sufficient one to single out a species.

However, some key cases must be considered. This compatible or viable (minor) diversity may suddenly become relevant, when ecosystemic changes modify the range of viability and enable the singularity of a few individuals to enhance specific differences. This may lead to speciation in all its forms (allopatric, peripatric, etc), often the result of rare events in individual migration, see (Zenik, 2015). In the numerous examples in (Venditti et al., 2009), "new species emerge from single events, each rare but individually sufficient to cause speciation". In most known cases, the permanent exploration of novelty, due either to genetic or ecosystemic instability or their interplay, leads to "hopeful monsters", in the broad sense of (Gould, 2002) - these are now understood as a complex blend of cumulative changes and punctual jumps, see (Chouard, 2010), that open new phylogenetic paths when enabled by a new or co-constructed ecosystemic niche. That is, the unavoidable variation proper to any reproduction, when it is particularly large, either by a cumulative effect or a sudden change, may generate a "monster" with the respect to the existing individuals. This may (hopefully) find its way in a changing ecosystem. Note that each hopeful monster, either viable or not, is a relatively rare event; most fail, but, in rare cases, they are enabled and speciate, possibly because enabled by rare environmental changes. One of the most famous examples in evolution, the evolutionary changes of Darwin's finches in the Galapagos, are now known to be related to rare and severe El Nino events that modified their food supply (Grant, 1993).

In this conceptual frame, historical time is then scanned by relevant, possibly rare changes in the phase space of evolution ${ }^{34}$. In some cases, though, speciation may happen within a list of pre-given

\footnotetext{
${ }^{33}$ As for the liver and in contrast to the immune systems and even lungs, in (Bravi, Longo, 2015), we naively accepted the claims of "noise biology", an approach that we criticize (randomness should be considered "noise”, in biology, to be analyzed by averaging out, central limits etc): in liver, we wrote, what only matters is the average enzymatic production. Yet, even in this case, the statistical, "averaging out” approach is wrong. That is, even such an apparently "dull” organ contributes to the organism's and to its own stability by an extensive aneupolidy and polyploidy of its cells (almost $50 \%)$. By this form of cells' diversity, the liver better responds to toxic injuries and damages: "subsets of aneuploid hepatocytes that are differentially resistant to the injury remain healthy, regenerate the liver and restore function” (Duncan, 2013).

${ }^{34}$ Rarity, though, may be historical or relative. In experimental evolution, in Echerichia coli, extremely rare mutations
} 
"symmetries" or possible phenotypes. This is the case, when peculiar niches enabled the survival of rare blind monsters and thus contributed to the formation of the 170 species of blind fishes (cavefishes). Similarly as for the kiwi bird (an apterygidae with no wings, see wikipedia). It seems to have reached New Zealand by flying. Then some hopeful monsters with no wings were enabled by an environment with no predators and an insect rich ground. Probably, the insect poor atmosphere made more viable the little running, clumsy bird, than its flying cousin, which is extinct.

In contrast to these cases, though, phenotypic complexity increases on average along evolution (Gould, 1996), according to the measure of complexity formalized in (Bailly, Longo, 2009). The cases of exaptation mentioned in section 4 and 7 correspond to increasing complexity and radically novel structures, such as internal hears' bones, feathers etc or ... the singular but sexually attractive festooned area on top of the reinforced shoulders bones of the Irish Elk, a new and unusual phenotype (Gould, 2002). Each of these cases is the result of rare events in phylogenesis: both the hopeful monster and an enabling context are rare. The rare combination of rare events form the branches or network of phylogenetic histories.

Note that this radically departs from common views in mathematical biology that mostly transfer conceptual frames and techniques from physics to life sciences, as observed also by tenants of a "physicalist" approach in biology (see footnote 24). As we observed in footnote 8, Réné Thom lucidly presents the philosophy still now guiding these analyses. More precisely, "the global landscape of the possible solutions ... preexists to the noise affecting the system". Thus a "fluctuation becomes relevant, but only in the frame of a pre-existing bifurcation". This is why, according to Thom, Prigogine's stress on noise (see Prigogine's papers in (Amsterdamski, 1990)) cannot be the focus of the scientific analysis: what matters is the pregiven "definition of all subgroups in which a given [group of] symmetry may break”, insists Thom (pp. 70-71). This may be the case for the blind fishes or the wingless birds, a symmetry change in a pre-given space, but not in the largely prevailing evolutionary changes that increase complexity by new phenotypes, as in the examples above of evolutionary exaptation.

In summary, fluctuations in non-linear physical dynamics cause the choice of a path or another within a pre-given list of possibilities. Moreover, when facing a bifurcation, a critical transition ... there is always a fluctuation and it will cause the dynamics to move along one or the other of the pre-given possible paths. As Thom says, the mathematical structure is already there: it is needed in order to write the equations. In an $n$-ary-furcation, the fluctuations may be partioned in $n$ equivalence classes of possibly different probabilities, each leading to one of the $n$ possible paths. In each on the $n$-partition, the fluctuations are generic and are far from rare. In an historical sciences instead, it is the diversity and rarity of new events that make history, because in a population, relevantly diverse organisms, which may contribute to speciation, are mostly rare. Moreover, they fail, most of the time; yet, rarely, they co-constitute with an enabling environment those possible paths that are not pre-given and thus change the phase space (by internal hears, feathers, wings from front podia ...). But ... how "rare" is a rare event?

\subsection{More on the frequency of rare events}

Well beyond the physics of noise, which refers to frequent but small fluctuations, a very interesting area of physics deals with "rare and large fluctuations", mostly situated in the tail of a distribution, thus with very low probabilities (Vulpiani et al., 2014; Bertini et al., 2015). This theory of large deviations in equilibrium and in far from equilibrium systems broadly enriches the traditional analysis of classical dynamics and statistical mechanics: it singles out the relevance of rare fluctuations, in pre-given phase spaces. These modify the probabilities of possible dynamics in an

unexpectedly turned out to be relatively frequent after a particular history of the population. In particular, their phenotypic expression may contingently depend on prior mutations in that population, along a 30,000 generations' history (Blount et al., 2008). Yet, according to these authors, it does not seem to be the result of gradual, cumulative changes: rare events may become frequent, after a long history in a rare environment (for E.c., an artificial glucoselimited medium that also contains citrate, (Blount et al., 2008)). 
unexpected and relevant way, far from averaging out or central limits. As a matter of fact, the probability distribution of small fluctuations or noise is always gaussian, following the central limit theorem, while, in large fluctuations, strong separation from the gaussian behavior is observed (Hurtado et al., 2013). More examples are given in (Kogan, 2014), where large fluctuations and rare events are closely analyzed in the tails of the probability distribution and their relevance is stressed. In a sense, these novel approaches go well beyond the so called Cournot's Principle, in physics: "It is a practical certainty that an event with very small probability will not happen” (and thus are not worth an analysis).

However, these singularities still belong to pre-listable sets of events or possible subgroups of a given group of symmetry, in a pre-defined phase space. In biology, instead, only a posteriori we can consider "possible" a not yet existing hopeful monster, or the result of an exaptation, a spandrel or one of the many rare forms and events that scan the historical time of the changing phase space of evolution. But then, if one cannot attribute probabilities to a phenotype not yet there, what does "rare" mean? Rarity is an a posteriori judgment, as most or all evolutionary judgments, such as "this phenotype is better than that". Similarly, a not yet existing phenotype may be a posteriori considered rare, within a diverse population, when its early occurrences and/or ecosystemic enablement conditions in time are a posteriori seen to be in small proportion. In other words, it is an a posteriori judgment concerning a new phenotype or an unpredictable success of an abnormal phenotype.

As for a further example of a rare evolutionary event along our lines, (Harms M., Thornton, 2014) first acknowledge that "it is difficult to know what happened in the deep past" in order to fully understand present biological functions, then reconstruct a rare bio-physical event in "the evolution of cortisol specificity in the ancestral glucocorticoid receptor (GR)". More examples can be found even in rather classical modeling approaches, such as the one in "Species survival emerge from rare events of individual migration” (Zenik, 2015).

The key difference between the physical analysis of relevant large fluctuations and rare events and the biological investigations of rare events and their evolutionary role may be summarized as follows:

In the few physical theories where rare events are analyzed, and not dumped at the margin of a Gaussian, it is considered rare that rare events relevantly shape physical dynamics. In biology instead, all evolutionary dynamics are shaped also by rare events.

Note that these biological events are rare, in the sense above, in view of their biological specificity or historicity: each event is individually rare, even if this type of events happens continually in evolution and contribute to the construction of all phylogenetic paths.

\section{0 - Conclusion}

Our tentative scientific understanding of a possible component of evolutionary novelty formation, or biological and cognitive "creativity", is inspired by the mathematical methodology in physics, where the notion of creativity makes no sense, or is at most a lousy name for randomness or unpredictability - while it may make sense in the dynamics of life systems. In these systems, we tried to understand novelty as the continual construction of new phase spaces, also on the grounds of re-interpreted traces of the past. The purpose is to contribute by our analysis to the construction of a rigorous scientific frame, in particular by unifying ontogenesis and phylogenesis (Longo et al., 2015; Soto, Longo, 2016), as a continuation of the endeavors in the books with Bailly and Montévil. The methodological, but "revisionist" transfer we operated here and in our books, from mathematics and physics towards biology, is an attempt to objectivize a common intuition: the joint role of inheritance and diversity production in evolution (and ontogenesis), as a "non-conservation" principle for phenotypes - inheritance is “descent with modification”, says Darwin. This goes 
beyond conservation principles (energy, momentum ...) and randomness in theories of the inert: it is compatible with them but extends them by new principles, which deal with new observables, that is, phenotypes and organisms.

Theoretical constructions must be based on frictions with "reality" that canalizes our endeavors towards knowledge. Observations, experiments and actions on nature provide these frictions and canalization, a knowledge praxis where Galileo and Darwin paved the way. The transfer of theories and techniques, as such, from (mathematical) physics to biology is an idealistic stand which grounds knowledge construction on pre-given conceptual frames, that is, on the transfer of "pure ideas", as belonging to a platonic, absolute realm. These ideas, instead, have been conceived through specific conceptual histories, in between mathematics and physics, and are not the result of the concrete investigations on the systems of life, with their proper phenomenality and empirical evidence. Unification is a further, difficult aim as physicists know very well, from Newton to Boltzmann, to the current researchers in the Quantum/relativistic interface, all at the origin of brand new mathematics.

We just made the explicit assumption, hopefully correct also for lack of a better approach, that, in biology, the construction of scientific objectivity could approximately follow the successful methodology of physics, even when facing a new object of knowledge, yet with no blind idealistic transfer of theories and techniques. We thus gave a central role to notions derived from mathematical invariants and their transformations and tried to adapt this method to the historicity and variability of life systems. We then developed one particular insight across disciplines, where the relevance of history, relational contexts and changes comes to the limelight of any empirical analysis. The common guideline of the different parts of this preliminary reflection is based on the role of the dynamical reconstruction of traces of the past in the understanding of the present and the shaping of the future in different systems of life, such as biology and cognition. The description of this phenomenon in one area is meant to help understanding it in the other, by a sense supporting network of different, but analogous conceptual constructions. It may also guide the transfer of this method to other historical sciences, as we did for economy concerning changing phase spaces (Felin et al., 2014; Koppl et al., 2015).

The conceptual abuses in this cross analysis are certainly numerous, from physics to biology and cognition. Yet, the shared approach, if just mildly correct, may provide the basis for closer investigations in each discipline. Recall that we incidentally transferred here also a negative result, such as the analysis of a form of unpredictability based on the relevance of the past and the difficulties in measuring it, which is a dual attitude if compared of the positivistic abuses of mathematical tools from physics in biology or even in describing and in ruling human, historical societies - as it too often happens in economy. As a matter of fact, our perspective implies also a radical critique of the very principles that are used in presumed scientific analyses of our social/historical dynamics, where, for example, totally unsuitable theory of (mathematical) equilibria still run the economy - unsuitable even for slightly complex physical systems. Our argument on the historicity of biology a fortiori applies to analyses of human life and history, where the creative interplay of memory and action is even stronger. That is, if we are right, the investigation of this form of unpredictability of the very space of possibilities, due also to the role of rare events and situated at the very level of the phase space and its historicity, may open the way to an understanding of a component of both evolutionary and human novelty creation. This is largely based on a context dependent re-use and re-interpretation of relatively invariant traces of the past, the historicized invariants.

Acknowledgment Claus Halberg and the referees made several insightful and constructive comments.

\section{References}


(Papers (co-)authored by Longo are downloadable from: $\underline{\text { http://www.di.ens.fr/users/longo/ ) }}$

Amsterdamski et al. (eds) La querelle du déterminisme, Gallimard, Paris, 1990.

Arndt, M., JuffmannTh., Vedral, V.. Quantum Physics meets Biology, HFSP Journal, Vol. 3, 6, 386-400, 2009.

Arnold V.-I., Catastrophe Theory, 3rd ed. Berlin: Springer-Verlag, 1992

Aspect A., Grangier P., Roger G., Experimental Realization of the Einstein-Podolsky-Rosen-Bohm

Gedankenexperiment : A New Violation of Bell's Inequalities, Phys. Rev. Let. 49, p.91, 1982.

Bailly F., Longo G.. Mathematics and the natural sciences; The Physical Singularity of Life. London: Imperial College Press, 2011 (original French version, Hermann, 2006).

Bailly, F., Longo, G.. Biological organization and anti-entropy. Journal of Biological Systems 17, 63-96, 2009.

Bécavin C, J.M. Victor \& A. Lesne The condensed chromatin fiber: an allosteric chemo-mechanical machine for signal transduction and genome processing Physical Biology 9, 013001 , 2012.

Berry, M.. Anticipations of Geometric Phase. Physics Today 43 (12): 34-40, 1990.

Berthoz A., The Brain Sens of Movement, Harvard U.P., 2000.

Bertini L., De Sole A., Gabrielli, D., Jona-Lasinio G., Landim C. Macroscopic fluctuation theory, arXiv:1404.6466 [cond-mat.stat-mech], Jan. 2015.

Binney J., Dowrick N.J. , Fisher A.J., Newman M.E.J., The Theory of Critical Phenomena: An Introduction to the Renormalization Group. Oxford U. P., 1992.

Bizzarri M., The New Alchemist. The Risks of Genetic Modification. WIT Press, Boston, 2012.

Blount, Z. D., Borland, C. Z. \& Lenski, R. E. Historical contingency and the evolution of a key innovation in an experimental population of Escherichia coli. Proc. Natl Acad. Sci. USA 105, 7899-7906, 2008.

Botzung A., Denkova E., Manning L. Experiencing and future personal events: Functional neuroimaging on the neural bases of mental time travel, Brain and Cognition, Volume 66, Issue 2, 202-212, March 2008.

Brentari C., Jakob von Uexküll: The Discovery of the Umwelt between Biosemiotics and Theoretical Biology, Springer, 2015.

Buiatti M., Longo G. Randomness and Multi-level Interactions in Biology. In Theory in Biosciences, vol. 132, n. 3:139-158, 2013.

Calude C. Information and randomness. Springer-Verlag, Berlin, second edition, 2002.

Calude C., Longo G. Classical, Quantum and Biological Randomness as Relative Incomputability. Invited Paper, special issue of Natural Computing, Springer, to appear, 2015.

Chibbaro S., Rondoni l., Vulpiani A. Reductionism, Emergence and Levels of Reality, Springer, Berlin, 2014.

Chouard T. Evolution: Revenge of the hopeful monster. Nature 463, 864-867, 2010.

Connes, A., Non-commutative Geometry, New York: Academic Press, 1994.

Deacon T., Incomplete Nature: How Mind Emerged from Matter. W.W. Norton \& Company, New York, 2011

Deacon T., Steps to a Science of Biosemiotics. Green Letters: Studies in Ecocriticisms. Online, August 5, 2015.

Desprat, N., Supatto, W., Pouille, P.-A., Beaurepaire, E., \& Farge, E.. Tissue deformation modulates twist expression to determine anterior midgut differentiation in Drosophila embryos. Developmental Cell, 15(3), 470-477, 2008.

Devaney R. L., An Introduction to chaotic dynamical systems, Addison-Wesley, 1989.

Disertori M., Sabot C. \& Tarrès P.. Transience of Edge-Reinforced Random Walk, Communications in Mathematical Physics, 339(1):121-148 , 2015.

Duncan AW., Aneuploidy, polyploidy and ploidy reversal in the liver. Semin. Cell Dev. Biol., Apr;24(4):347-56, Jan 16, 2013.

Edelman G., The Remembered Present: A Biological Theory of Consciousness. New York: Basic Books, 1990.

Edelman G. M. et Gally J. A., Degeneracy and Complexity in biological systems, Proceedings of the National Academy of Science, 24, 13763-13768, 2001.

Edelman G., Tononi G. A Universe of Consciousness. How Matter Becomes Immagination, Basic Books, 2000.

Einstein A., Podolsky B. Rosen N., 1935, Can Quantum-Mechanical Description of Physical Reality be Considered complete?, Phys. Rev., 41, 777.

Felin T., S. Kauffman, R. Koppl, G. Longo. Economic Opportunity and Evolution: Beyond Bounded Rationality and Phase Space. In "Strategic Entrepreneurship Journal", vol. 8, issue 4: 269-282, 2014.

Fernandez-Sanchez, M.-E., Serman, F., Ahmadi, P., \& Farge, E.. Mechanical induction in embryonic development and tumor growth integrative cues through molecular to multicellular interplay and evolutionary perspectives. Methods in Cell Biology, 98(10), 295-321, 2010.

Gil F., Il tempo del pensiero, in Le frontiere del tempo (Romano ed.) Il Saggiatore, Milano, 1981.

Gogarten, JP; Townsend, JP. "Horizontal gene transfer, genome innovation and evolution.". Nature reviews. Microbiology 3 (9): 679-87, 2005.

Goldenfeld N., Woese C. Life is Physics: Evolution as a Collective Phenomenon Far From Equilibrium, Ann. Rev. Condens. Matter Phys. 2:375-399, 2011.

Gould S.-J., Wonderful Life, Norton \& Co., 1989.

Gould S.-J., E. Vrba, Exaptation - a missing term in the science of form, Paleobiology 8, p. 4-15, 1982. 
Gould, S.J., Full House. Three Rivers Press, New York, 1996.

Gould S.-J., The Structure of Evolutionary Theory, Harvard U. Press, 2002.

Grafen A., The formal darwinism project in outline, Biol Philos DOI 10.1007/s10539-013-9414, 1982.

Grant B., Grant P., Evolution of Darwin's finches caused by a rare climatic event. Proc. R. Soc. London, 251, 111-117, 1993.

Harms M., Thornton J., Historical contingency and its biophysical basis in glucocorticoid receptor evolution, Nature 512, 203-207, 2014.

Hurtado, P. I., Lasanta A., Prados A., Typical and rare fluctuations in nonlinear driven diffusive systems with dissipation, Phys. Rev. E 88, 022110, 2013.

Huxley, J., 1943, Evolution, the modern synthesis, Harper and Brothers Publishers, New York and London.

Kauffman S.A., Investigations, Oxford University Press, USA, 2002.

Keeling P.J., Palmer J.D.. Horizontal gene transfer in eukaryotic evolution. Nat. Rev. Genet. 9(8):605-18, 2008.

Kogan O., Onset of Singularities in the Pattern of Fluctuational Paths of a Nonequilibrium System, in arXiv. org, http://arxiv.org/abs/1110.2820, Oct 2014 (see also https://sites.google.com/site/olegkogansite/fluctuations )

Koppl R., S. Kauffman, G. Longo, T. Felin. Economy for a Creative World. Target article "Journal of Institutional Economics", Vol. 11, Issue 01, pp 1 - 31, March 2015.

Kosmann-Schwarzbach Y.. The Noether theorems: Invariance and conservation laws in the twentieth century. Springer-Verlag, Berlin, 2010.

Islam J. N., An Introduction to Mathematical Cosmology, Cambridge U.P., 2001.

Jablonka E, Lamb MJ. 1, Evolution in Four Dimensions, MIT Press, 2008.

Jablonka E, Lamb MJ.. Epigenetic inheritance in evolution. J. Evol. Biol. 11(2):159-83, 1998.

Jacob P., Le Jeu des possibles, essai sur la diversité du vivant, Fayard, 1981.

Laskar J. "Large scale chaos in the Solar System", Astron. Astrophys., 287, L9 L12, 1994.

Lemke H., Antonio Coutinho and Hans Lange, Lamarckian inheritance by somatically acquired maternal IgG phenotypes, TRENDS in Immunology Vol.25 No.4, April 2004

Lesne A., Robustness: confronting lessons from physics and biology, Biol Rev Camb Philos Soc., Nov. 83(4): 509-32, 2008.

Longair M., The Cosmic Century: A History of Astrophysics and Cosmology, Cambridge U. Press, 2006.

Longo, G. Interfaces of Incompleteness. Italian version in La Matematica, vol. 4, Einuadi, Torino, 2010 (downloadable in English).

Longo G., Conceptual Analyses from a Grothendieckian Perspective: Reflections on Synthetic Philosophy of Contemporary Mathematics by Fernando Zalamea, in Speculations, December 2015 (https://www.urbanomic.com/speculations lo/).

Longo G., Montévil M., Kauffman S., 2012, No entailing laws, but enablement in the evolution of the biosphere. Invited Paper, ACM proceedings of the Genetic and Evolutionary Computation Conference, GECCO’12, Philadelphia (PA, USA), July 7-11, 2012.

Longo G., Montévil M.. Perspectives on Organisms: Biological Time, Symmetries and Singularities. Dordrecht: Springer, 2014.

Longo G., Montévil M.. Models vs. Simulations: a comparison by their Theoretical Symmetries. To appear in Springer Handbook of Model-Based Science, 2015.

Longo G, Montévil M., Sonnenschein C. Soto A. In Search of Principles for a Theory of Organisms. Journal of Biosciences, to appear, 2015.

Longo G., M. Mugur-Schachter (Editors) Developments of the Concepts of Randomness, Statistic, and Probability", Special issue of Mathematical Structures in Computer Science, Cambridge University Press, vol.24, n.3, 2014.

Longo G., Perret N., Contributions to a Theory of Biological Time: Anticipation, Protention and Biological Inertia. In Building Theories, Sciences and Hypotheses (Ippoliti, ed.), Springer, 2017.

Marinucci A. From deterministic biology to relational biology, in preparation, 2017.

Miquel P.A. Sur le concept de nature, Hermann, Paris, 2015.

Misslin R.. Une vie de cellule. Forme et espace. Dans "Géométrie et Cognition", G. Longo (Editeur), numéro spécial de la Revue de Synthèse, Editions de la rue d'Ulm, tome 124, 2003-4.

Montévil M. Mossio M. Closure of constraints in biological organisation, in Journal of Theoretical Biology, vol. 372: 179-191, 2015.

Moreno A., Mossio M., Biological Autonomy, a philosophical and theoretical enquire, Springer, 2015.

Needham, J., 'Human Laws and the Laws of Nature in China and the West', Journal of the History of Ideas, vol. XII, 3-32, 194-231, 1951

Nicolis G., I. Prigogine. Self-organization in non-equilibrium systems. New York, Wiley, 1977.

Nowacki M, Landweber LF. 2009. Epigenetic inheritance in ciliates. Curr. Opin. Microbiol. 12(6):638-43.

Paby A., Rockma M., Cryptic genetic variation: evolution's hidden substrate, Nature Reviews Genetics, 15, 247258 (2014) 
Pal C, Papp B, Lercher MJ.. Adaptive evolution of bacterial metabolic networks by horizontal gene transfer. Nat. Genet. 37(12):1372-75, 2005.

Plankar M., Jerman I, Krasovec R. On the origin of cancer: can we ignore coherence?, Progr. Biophys. Mol. Biology, vol. 106, 2, August, 2011.

Prochiantz A. Les anatomies de la pensee, Odile Jacob, 1997.

Rando OJ, Verstrepen KJ.. Timescales of genetic and epigenetic inheritance. Cell 128(4):655-68, 2007.

Roux, S., “Controversies on Legality (1680-1710)”, in Natural Law and Laws of Nature in Early Modern Europe, éd. L. Daston et M. Stolleis, Aldershot, Ashgate Pub., p. 199-214, 2009.

Rowan S., Hough J., Crooks D. Thermal noise and material issues for gravitational wave detectors Physics Letters A, Volume 347, Issues 1-3, Pages 25-32, 14 November 2005.

Schwartz, L., Théorie des distributions 1-2, Hermann, 1951.

Shomrat T., Levin M., An automated training paradigm reveals long-term memory in planarians and its persistence through head regeneration, The Journal of Experimental Biology 216, 3799-3810, 2013.

de Souza F., Franchini L., Rubistein M., Exaptation of Transposable Elements into Novel Cis-Regulatory Elements: Is the Evidence Always Strong?, Mol Biol Evol.; 30(6): 1239-1251, June 2013.

Soto A., Longo G. (eds.) A Theory of Organisms, Special issue of Progress in Biophysics and Molecular Biology, Elsevier, 2016, <10.1016/j.pbiomolbio.2016>.

Szpunar K., Watson J., McDermott K., Neural substrates of envisioning the future, PNAS, 104, 2, 643-647, Jan. 2007.

Torday J.-S.. What We Talk About When We Talk About Evolution. Cell Communication Insights:7, 1-15, 2015.

Uzan J-P., Varying Constants, Gravitation and Cosmology, Living Reviews in Relativity 14(2), 1-155, 2011.

Venditti C., Meade A., Pagel M. Phylogenies reveal new interpretation of speciation and the Red Queen. Nature 463, 349-352, 21 January 2010.

Vulpiani A., Cecconi F., Cencini M., Puglisi A., Vergni D. (editors), Large Deviations in Physics , Springer, 2014.

Weyl H., Philosophy of Mathematics and of Natural Sciences. Princeton U. Press, 1949.

Zalamea F., Synthetic Philosophy of Contemporary Mathematics, Urbanomic and Sequence Press, NY, 2012.

Zenik Y., Solomon S., Yaari G., Species survival emerge from rare events of individual migration, Nature Scientific Reports 5, Article number: 7877, 2015.

West-Eberhard M-J.. Developmental plasticity and evolution. Oxford University Press, New York, 2003.

West-Eberhard M-J.. Developmental plasticity and the origin of species differences, PNAS, May 3, vol. 102, suppl. 1, 6543- 6549, 2005. 